\title{
Mars Ion and Neutral Particle Analyzer (MINPA) for Chinese Mars Exploration Mission (Tianwen-1): Design and ground calibration
}

\author{
LingGao Kong1,2,3,4* , AiBing Zhang 1,2,3,4* Zhen Tian 1,2,3,4, XiangZhi Zheng 1,2,3, WenJing Wang 1,2,3,4, Bin Liu 1,2,3, \\ Peter Wurz ${ }^{5}$, Daniele Piazza ${ }^{5}$, Adrian Etter5, Bin Su1,2,3, YaYa An 1,2,3, JianJing Ding 1,2,3, WenYa Li1,6, Yong Liu1,6, \\ Lei Li,6, YiRen Li7, Xu Tan ${ }^{\mathbf{8}}$, and YueQiang Sun 1,2,3,4 \\ ${ }^{1}$ National Space Science Center, Chinese Academy of Sciences, Beijing 100190, China; \\ ${ }^{2}$ Beijing Key Laboratory of Space Environment Exploration, Beijing 100190, China; \\ ${ }^{3}$ Key Laboratory of Science and Technology on Space Environment Situational Awareness, Chinese Academy of Sciences, Beijing 100190, China; \\ ${ }^{4}$ University of Chinese Academy of Sciences, Beijing 100049, China; \\ 5Physics Institute, University of Bern, Bern 3012, Switzerland; \\ ${ }^{6}$ State Key Laboratory of Space Weather, Beijing 100190, China; \\ 7 University of Science and Technology of China, Hefei 230026, China; \\ ${ }^{8}$ National Astronomical Observatories, Chinese Academy of Sciences, Beijing 100012, China
}

Citation: Kong, L. G., Zhang, A. B., Tian. Z., Zheng, X. Z., Wang, W. J., Liu, B., Wurz, P., Piazza, D., Etter, A., Su, B., An, Y. Y., Ding, J. J., Li, W. Y., Liu, Y., Li, L., Li, Y. R., Tan, X. and Sun Y. Q. (2020). Mars lon and Neutral Particle Analyzer (MINPA) for Chinese Mars Exploration Mission (Tianwen-1): Design and ground calibration. Earth Planet. Phys., 4(4), 333-344. http://doi.org/10.26464/epp2020053

\begin{abstract}
The main objective of the Mars lon and Neutral Particle Analyzer (MINPA) aboard the Chinese Mars Exploration Mission (Tianwen-1) is to study the solar wind-Mars interaction by measuring the ions and energetic neutral atoms (ENAs) near Mars. The MINPA integrates ion and ENA measurements into one sensor head, sharing the same electronics box. The MINPA utilizes a standard toroidal top-hat electrostatic analyzer (ESA) followed by a time of flight (TOF) unit to provide measurement of ions with energies from $2.8 \mathrm{eV}$ to $25.9 \mathrm{keV}$ and ENAs from $50 \mathrm{eV}$ to $3 \mathrm{keV}$ with a base time resolution of 4 seconds. Highly polished silicon single crystal substrates with an $\mathrm{Al}_{2} \mathrm{O}_{3}$ film coating are used to ionize the ENAs into positive ions. These ions can then be analyzed by the ESA and TOF, to determine the energy and masses of the ENAs. The MINPA provides a $360^{\circ} \times 90^{\circ}$ field of view (FOV) with $22.5^{\circ} \times 5.4^{\circ}$ angular resolution for ion measurement, and a $360^{\circ} \times 9.7^{\circ} \mathrm{FOV}$ with $22.5^{\circ} \times 9.7^{\circ}$ angular resolution for ENA measurement. The TOF unit combines a $-15 \mathrm{kV}$ acceleration high voltage with ultra-thin carbon foils to resolve $\mathrm{H}^{+}, \mathrm{He}^{2+}, \mathrm{He}^{+}, \mathrm{O}^{+}, \mathrm{O}_{2}{ }^{+}$and $\mathrm{CO}_{2}+$ for ion measurement and to resolve $\mathrm{H}$ and $\mathrm{O}(\geq 16$ amu group) for ENA measurement. Here we present the design principle and describe our ground calibration of the MINPA.
\end{abstract}

Keywords: Mars; ion; energetic neutral atom; electrostatic analyzer; time of flight

\section{Introduction}

Unlike Earth, Mars lacks a global intrinsic magnetic field to shield its atmosphere from the solar wind. The histories of the Martian atmosphere and its liquid water are related directly to the solar wind-Mars interaction mechanism which has thus been a main scientific objective since the very beginning of human exploration of Mars, and is still not fully understood. Since the 1960s, successful Mars missions - for example, Mariner 4, Mars 2/3, Viking 1/2, Phobos-2, MGS, Mars Express, and MAVEN (Neugebauer and Snyder, 1962; Farmer et al., 1977; Verigin et al., 1991; Mitchell et al., 2001; Barabash et al., 2006; McFadden et al., 2015 - have led to great progress in the research areas of solar wind-Mars interac-

Correspondence to: L. G. Kong, klg@nssc.ac.cn

$$
\text { A. B. Zhang, zhab@nssc.ac.cn }
$$

Received 26 MAY 2020; Accepted 16 JUN 2020.

Accepted article online 22 JUL 2020.

C 2020 by Earth and Planetary Physics. tion and the evolution of the Martian atmosphere. Particle and field instruments have always been selected as key scientific payloads of Mars missions. The Mars atmosphere is ionized by solar wind and ultraviolet (UV) light to create a plasma environment around the planet (its ionosphere and induced magnetosphere). Without the shielding of a magnetic field, ions can easily escape from the Mars system. The planet's ion loss rate is critical to understand the solar wind-Mars interaction and the evolutionary history of the planet's atmosphere. Commonly used techniques to investigate the interaction and estimate the ion loss rate have been in situ ion measurements and remote imaging using energetic neutral atoms (ENA) (Barabash et al., 2006). The in situ ion measurement approach provides a more accurate ion distribution function. ENA imaging provides a global two-dimensional image of the interaction volume (Wurz, 2000; McKenna-Lawlor et al., 2005).

Ion mass spectrometers have been widely used in previous Mars 
missions to detect in situ the ion environment around Mars and to estimate the ion loss rate. Nevertheless, the estimates of ion loss rate from several previous successful missions (Lundin et al., 1990; Carlsson et al., 2006; Barabash et al., 2007; Fang XH et al., 2010; Ramstad et al., 2013, Ledvina et al., 2017) have exhibited significant disagreement on this point. Thus, it is of great importance that the next generation of Mars missions include ion measurements of significantly improved precision. The solar wind upstream of the Mars' bow shock can experience charge exchange with the Martian hydrogen exosphere to produce ENAs. Such ENAs were first detected in Earth orbit by the Low Energy Neutral Atom (LENA) instrument (Moore et al., 2000) onboard the terrestrial magnetospheric Imager for Magnetopause-to-Aurora Global Exploration (IMAGE) mission (Moore et al., 2001; Collier et al., 2001). The first ENA measurements in Mars orbit were carried out by the Neutral Particle Imager (NPI) and the Neutral Particle Detector (NPD) onboard the orbiter of the Mars Express mission (Barabash et al., 2006; Lundin et al., 2009). The ASPERA-3/NPD instrument has collected ENA data in the Martian dayside atmosphere, in the planet's magnetosheath, and in the magnetotail of its magnetosphere (Futaana et al., 2006; Gunell et al., 2006; Galli et al., 2008).

The Chinese Mars Exploration Mission (Tianwen-1) consists of an orbiter, a lander, and a rover. The main scientific objectives of the orbiter are: (1) comprehensive investigation of the Martian space environment, the induced magnetosphere and the ionosphere; (2) precise exploration of the Martian gravity field and atmosphere; (3) mapping of Martian surface topography and efficient observation of sandstorms; and (4) survey of rock composition, soil characteristics, and distribution of water ice and minerals on the surface of Mars (Dongfang X, 2016; Ye PJ et al., 2017). The orbiter will collect data while in an orbit of $265 \mathrm{~km} \times 9600 \mathrm{~km}$ with inclination angle of $93^{\circ}$.

The Mars lons and Neutral Particles Analyzer (MINPA) is one of the seven scientific payloads on the orbiter. The main objective of the MINPA is to study the solar wind-Mars interaction by measuring ions and energetic neutral atoms (ENAs), in tandem with simultaneous magnetic field measurements by a magnetometer onboard the orbiter. As shown in Figure 1, the MINPA is mounted on one of the corners of the $+X$ panel of the spacecraft. The electronics box is under the deck and the sensor head protrudes out of the $+X$ panel. The green hemisphere indicates the $2 \pi$ FOV of the ion

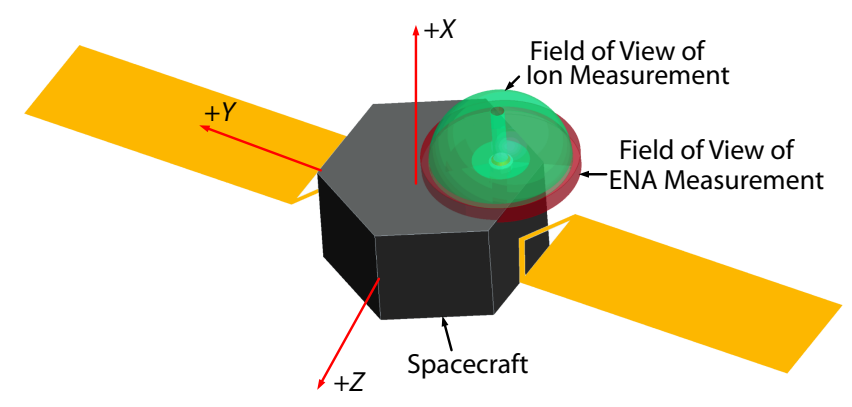

Figure 1. Layout of the MINPA on the orbiter, in the right-hand spacecraft coordinate system. The green hemisphere and the red torus denote the FOVs of the ion and the ENA measurements, respectively. measurement, while the red torus below the green hemisphere denotes the FOV of the ENA measurement. During the interplanetary cruise phase, from the Earth to Mars, the $+X$ direction is toward the Sun. In the Mars orbit, $+Z$ indicates the direction toward the center of Mars when the altitude is below $800 \mathrm{~km}$, and away from the Sun when the orbiter altitude is above $800 \mathrm{~km}$.

\section{MINPA Design}

The MINPA consists of two units: the ion and ENA units. These two units are integrated into one sensor head and share one electronics unit. Figure 2 shows a cross-sectional view of a functional diagram of the MINPA. The sensor head consists of one ion measurement channel and one ENA measurement channel, each with its own entrance window. The symmetry axis, the azimuth angle $(\theta)$, and elevation angle $(\varphi)$ of the sensor FOV are also illustrated in Figure 2.

For the ion measurement channel, external ions in the space environment are selected along the elevation angle by the deflection system at the entrance (deflector 1 and deflector 2 electrodes). The incidence elevation angle, which varies from $0^{\circ}$ to $90^{\circ}$, is controlled by the high voltage differential applied to the two deflectors 1 and 2 . The ion trajectories are then adjusted by the deflector 3 so that the ions can enter the toroidal top-hat electrostatic analyzer (ESA).

For the ENA measurement channel, the ENAs enter the sensor head from the side, around a central elevation angle of $\sim 0^{\circ}$. Before the ENAs reach the charge exchange plates, a deflection system (deflector 4 and deflector 5 electrodes) is used to sweep away all the $\leq 60 \mathrm{keV}$ charged particles coming from the same viewing direction. The deflectors 4 and 5 also collimate the ENA beams to within an elevation angle range of $\sim 15^{\circ}$. The charge exchange plates, made from $25 \mu \mathrm{m} \mathrm{Al}{ }_{2} \mathrm{O}_{3}$ film coated on highly polished silicon single crystal substrates, were provided by the University of Bern, which supplied similar plates to the BepiColumbo mission (Riedo, 2010). The ENAs' incident angle on the charge exchange plates is approximately $15^{\circ}$, which permits the highest ionization efficiency (Barabash et al., 2006; Scheer et al., 2009). The positive particles ionized by the charge exchange plates are accelerated by the negative voltages of the extraction lens (lens 1 and 2 electrodes) and then enter the ESA.

The ion and ENA measurement channels share the same ESA and Time of Flight (TOF) unit in the sensor head. The ions from outer space and ions ionized by the charge exchange plates enter the ESA at different operation times, determined by the high voltages set in the sensor head, as follows: When the high voltages are applied on the charge exchange plates, lenses 1 and 2 are set to be 0 $\mathrm{V}$, the ENA measurement channel is switched off, and the ion measurement channel is switched on. When deflector 1 is set to be $0 \mathrm{~V}$ and deflectors 2 and 3 are set to the maximum negative voltage $(-6 \mathrm{kV})$, the ion measurement channel is switched off and the ENA measurement channel is switched on. To sweep away the charged particles from outer space, the voltages on deflectors 4 and 5 are always set to be +5 and $-5 \mathrm{kV}$, respectively.

The ESA is a toroidal top-hat electrostatic analyser that has been used in many previous space physics missions (Carlson and Mc- 


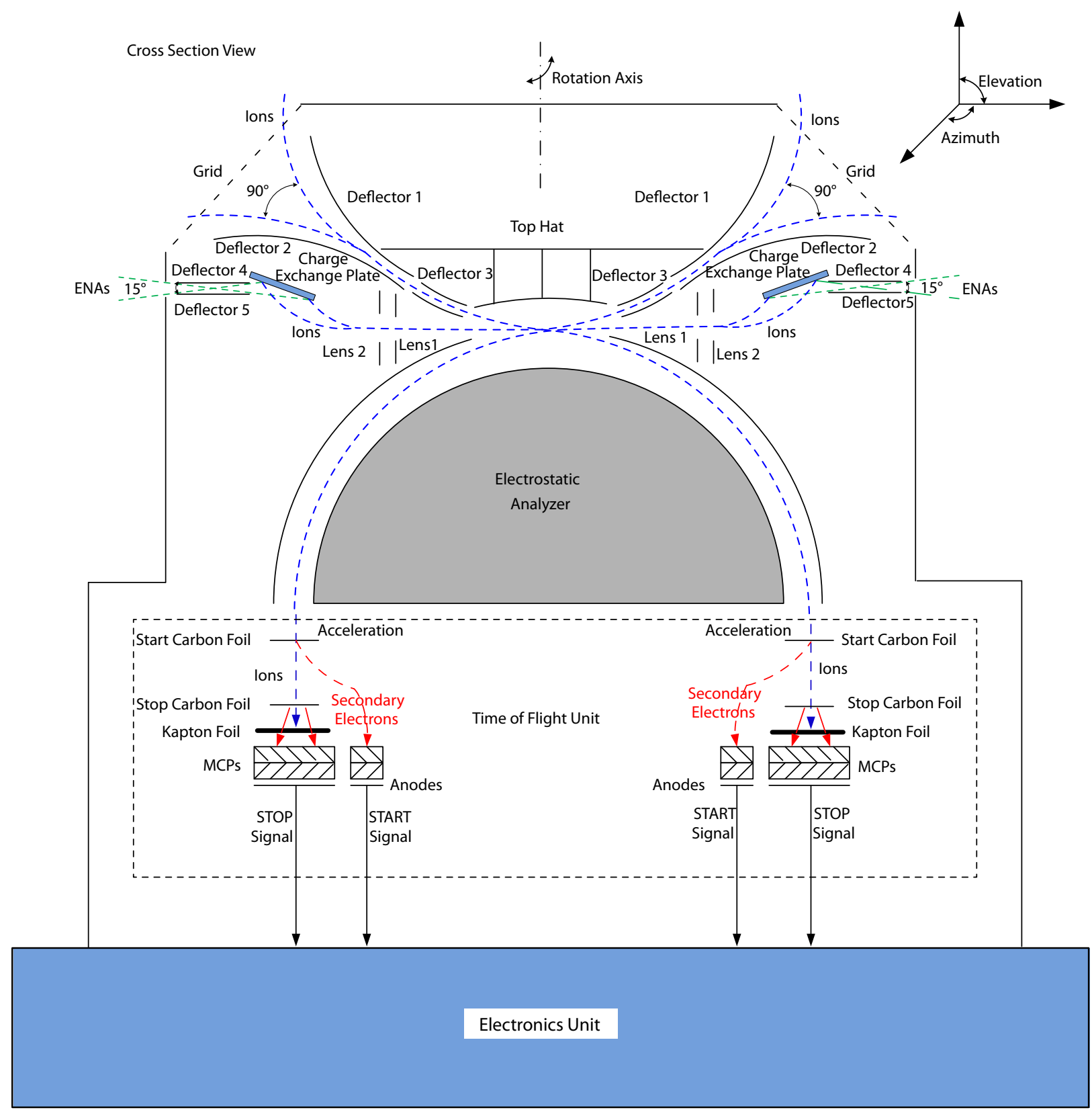

Figure 2. Cross-sectional view of the MINPA functional diagram. Nominal trajectories of ions, ENAs, and secondary electrons are shown in blue, green, and red dash curves, respectively. The definitions of azimuth and elevation angles in the instrument coordinate system are shown in the upper right corner.

Fadden, 1998; Klumpar et al., 2001; Rème et al., 1997, 2001, 2005). The electric field inside the ESA selects and deflects ions with a particular energy-per-charge. These ions are post-accelerated by a high voltage of $-15 \mathrm{kV}$ applied on the shell of the TOF, and enter the TOF where the velocities of different ion species can be analysed. The ESA is designed to shift the electrostatic focal point out of the exit in order to coordinate with TOF optics and thus achieve a mass measurement of relatively high resolution.

To measure ion velocities, the TOF unit needs to determine the START and STOP signals for a given flight distance. The TOF unit of the MINPA uses ultrathin carbon foils to generate these START and STOP signals. The START signal is triggered by the secondary electrons produced when the ions pass through the start carbon foil $\left(\sim 1 \mu \mathrm{g} / \mathrm{cm}^{2}\right)$; these electrons hit the START micro-channel plates (MCPs). The STOP signal is triggered by the secondary electrons produced when the ions pass through the stop carbon foil $\left(\sim 1 \mu \mathrm{g} / \mathrm{cm}^{2}\right)$; they hit the STOP MCPs. We use a thick foil $(50 \mathrm{~nm} \mathrm{Al}$ coated on $500 \mathrm{~nm}$ Kapton foil) inserted between the stop carbon foil and MCPs to stop the ions and neutrals and let only the secondary electrons pass through and hit the STOP MCPs. The stop carbon foil and thick foil are used to suppress ghost peaks, a topic discussed in more detail in the MAVEN STATIC instrument paper (McFadden et al., 2015).

The MINPA detects the secondary electrons from start and stop carbon foils with Z-stack 40:1 (thickness: channel diameter ratio) MCPs manufactured by the Photonis company in France. The 
MCPs bias voltage is software-controlled; the gain can be monitored by an adjustable threshold in the detection electronics.

When the secondary electrons generated from the START and STOP carbon foils hit the MCPs they produce charge pulses that are collected by 16 start and 16 stop discrete anodes, respectively.

The start and stop discrete anodes form two independent anode chains linked by 2 ns discrete delay line chips; each anode chain uses 15 delay line chips. Each delay line chip connects two adjacent anodes. The charge pulse events from the start or stop anodes split and propagate to both ends of the delay line. Four A121 preamplifiers are used for the two delay lines to shape and generate START signal A, START signal B, STOP signal A, and STOP signal $B$; this is shown schematically in Figure 3.

The four signals from the preamplifiers are fed to the time-to-digital-converter (TDC) board to produce four timing outputs, T_STA and T_STB for the START signals, and T_SPA and T_SPB for the STOP signals. These timing outputs are used to evaluate event times-of-flight (TDC1 $=$ T_STA $-\mathrm{T} \_S P A$ and TDC2 $=$ T_STB $-T$ _SPB). Both the TDC1 and the TDC2 are used for the event correlation statistics.

The event azimuth position can be determined from the timing difference of the START signals (TDC3 = T_STA-T_STB) or STOP signals (TDC4 = T_SPA-T_SPB), which are measured by a one field-programmable gate array (FPGA) chip. The redundant azimuth position determination allows rejection of events when the start and stop locations do not match due to angular scattering of electrons in the foil. The redundant time of flight outputs allow selection of the timing circuit with the smallest propagation delay, and therefore slightly improve the timing resolution.

The high voltages for the sensor head are listed in Table 1; they are generated by the high voltage power supply board in the electronics box. The electronics box also contains one FPGA board

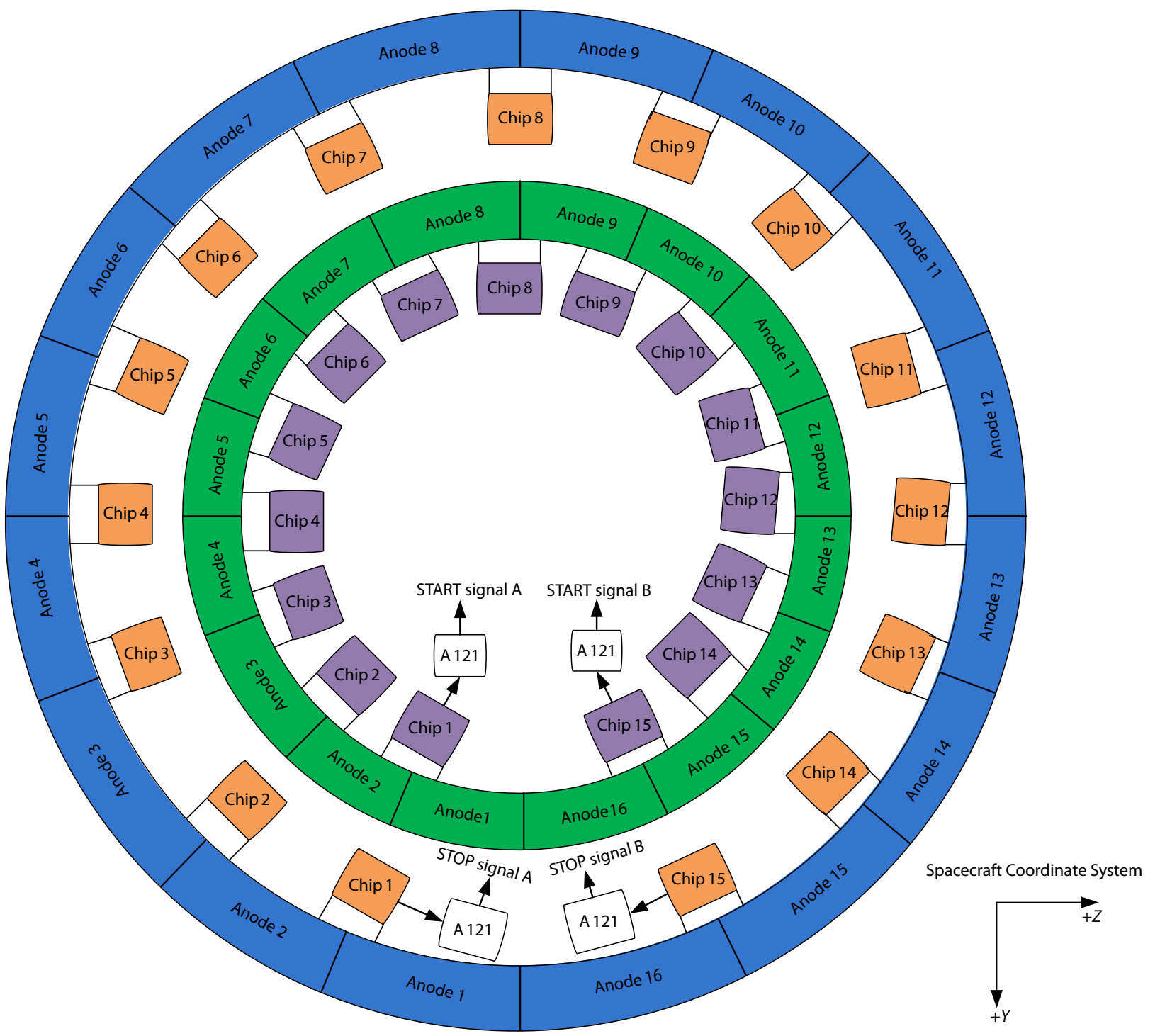

Figure 3. Top view of the diagram of the START and STOP delay line chains and the connection with the start and stop anodes. The 16 green inner sectors and 16 blue outer sectors are the start anodes and the stop anodes. The 16 purple and 16 orange blocks show the delay line chips for the START and STOP delay line chains, respectively. 
Table 1. High voltages setting in the MINPA sensor head.

\begin{tabular}{ccc}
\hline Items & High voltage range & Comment \\
\hline Deflector 1 & from 0 to $-6 \mathrm{kV}$ & Adjustable, sweep during operation \\
$\begin{array}{c}\text { Deflector } 2 \\
\text { feflector } 3\end{array}$ & from 0 to $-6 \mathrm{kV}$ & Adjustable, sweep during operation \\
Deflector 4 & from 0 to $+5 \mathrm{kV}$ & Adjustable, sweep during operation \\
Deflector 5 & from 0 to $-5 \mathrm{kV}$ & Adjustable, fixed at $+5 \mathrm{kV}$ during operation \\
Charge exchange plate & from 0 to $+5 \mathrm{kV}$ & Adjustable, fixed at $-5 \mathrm{kV}$ during operation \\
Lens 1 & from 0 to $-5 \mathrm{kV}$ & Adjustable, sweep during operation \\
Lens 2 & from 0 to $+5 \mathrm{kV}$ & Adjustable, sweep during operation \\
ESA & from 0 to $-5 \mathrm{kV}$ & Adjustable, sweep during operation \\
TOF shell & from 0 to $-15 \mathrm{kV}$ & Adjustable, sweep during operation \\
Thick foil & from 0 to $-4 \mathrm{kV}$ & Adjustable, fixed at $-15 \mathrm{kV}$ during operation \\
MCPs & from 0 to $-4 \mathrm{kV}$ & Adjustable, fixed at $-2.2 \mathrm{kV}$ during operation, maybe changed after in-flight calibration \\
\hline
\end{tabular}

to control the operation of the instrument and one low voltage power supply board to generate all the internal voltages for the instrument.

In general operation, the MINPA can have a maximum of 64 energy sweeping steps, 16 elevation angle deflection steps, and 16 azimuth sectors (16 anodes) for the ion measurement channel. It has 8 energy seeping steps and 16 azimuth sectors ( 16 anodes) for the ENA measurement channel. The working tables stored in the instrument define the energy and elevation angle sweeping steps by high voltage settings. The MINPA flight model is displayed in Figure 4.

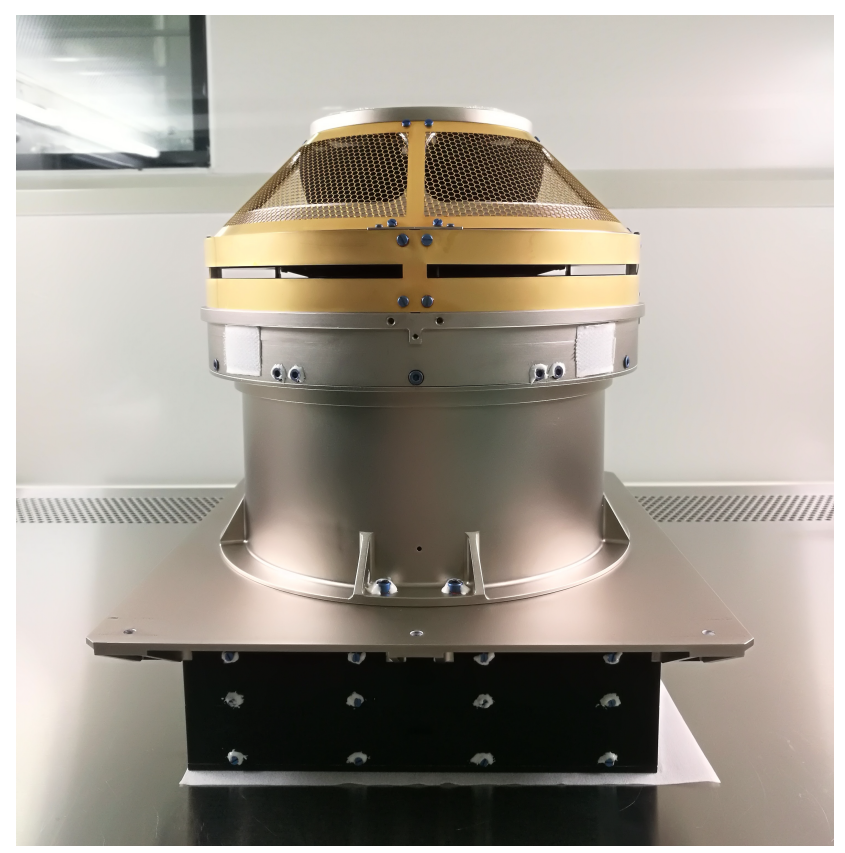

Figure 4. Flight model of MINPA.

\section{MINPA Ground Calibration}

Ground calibration of the MINPA was performed both in the MEFISTO (MEsskammer für FlugzeitInStrumente und Time-Of-
Flight) calibration chamber at the University of Bern and in the CASSPI (Calibration System for Space Plasma Instrument) calibration chamber at the National Space Science Center, Chinese Academy of Sciences. Figure 5 shows the MINPA in the MEFISTO and CASSPI calibration chambers. An ion beam, an ENA beam, and a UV light beam in MEFISTO were used for the MINPA calibration. The ion beam is a pencil beam produced by an electron-cyclotron resonance source; the beam energy can be adjusted between $10 \mathrm{eV} / \mathrm{q}$ and $100 \mathrm{keV} / \mathrm{q}$. The ENA beam in MEFISTO is produced by the interaction of an ion beam with a charge exchange surface in the Neutralizer (Wieser and Wurz, 2005). The UV light beam is an Ar excimer lamp with peak emission centered at $126 \mathrm{~nm}$.

Limited by the elevation angle of the turntable in the MEFISTO chamber, the calibration in MEFISTO cannot cover the whole MINPA FOV. The full FOV calibration for ion measurement was carried out in the CASSPI chamber. The ion beam in CASSPI is produced by a duo-plasmatron ion source. The ion beam has a diameter of $70 \mathrm{~mm}$ and can be adjusted between $100 \mathrm{eV} / \mathrm{q}$ and 30 $\mathrm{keV} / \mathrm{q}$. Helmholtz coils are used outside the vacuum chamber to reduce the inside background geomagnetic field strength to approximately $500 \mathrm{nT}$ in the center area of the chamber. A 5-axes turntable is mounted inside the chamber with 3 axes of translation and 2 axes of rotation to cover a $360^{\circ}$ (azimuth) $\times \pm 90^{\circ}$ (elevation) angular range.

\subsection{Calibration of the Ion Measurement Channel}

The MINPA responses to beams of $\mathrm{H}^{+}, \mathrm{C}^{+}, \mathrm{O}^{+}, \mathrm{H}_{2} \mathrm{O}^{+}, \mathrm{CO}^{+}, \mathrm{O}_{2}{ }^{+}$and $\mathrm{CO}_{2}{ }^{+}$at different energies were calibrated in the MEFISTO and CASSPI chambers. Figure 6 shows the response of MINPA's 16 azimuth anodes to $\mathrm{H}^{+}$beams with an energy of $10 \mathrm{keV}$ under a fixed elevation angle of $0^{\circ}$. As the turntable moved azimuthally from $0^{\circ}$ to $360^{\circ}$, the ion beam illumined anodes 1 to 16 , one by one. The curves from left to right in Figure 6 denote the response profiles of anode 1 to anode 16 . The turntable was rotated to cover $360^{\circ}$ within a very short time ( 3 minutes), during which the beam flux can be considered as constant. Therefore, the response profile of each anode has a few data points and is sharper than it should be. Numerical simulation implies that each anode has a FOV of about 


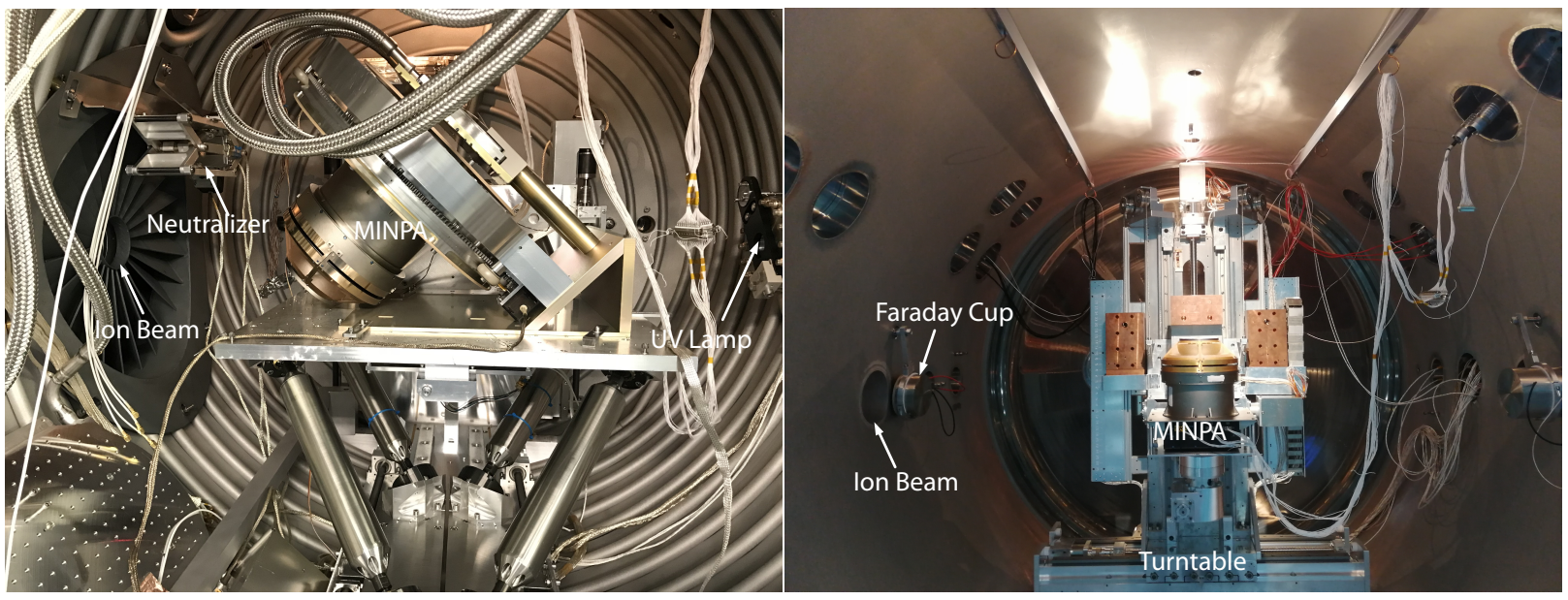

Figure 5. MINPA ground calibration of flight model in MEFISTO (left) and in CASSPI (right).

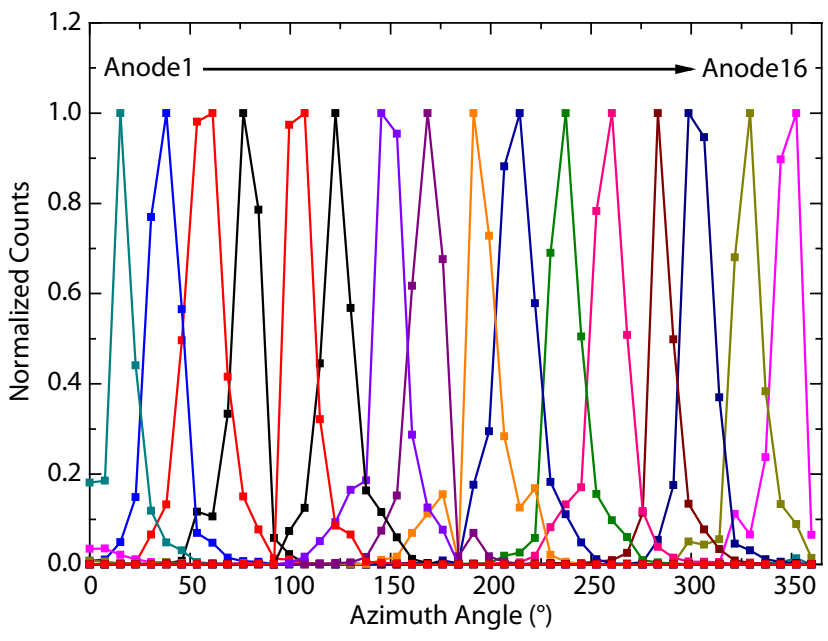

Figure 6. Response of 16 azimuth anodes to $10 \mathrm{keV} \mathrm{H}^{+}$, from the MINPA ground calibration in MEFISTO.

$22.5^{\circ}$ (full width at half maximum, FWHM) on average. The inside spokes of the MINPA's support structure, located at azimuth angles of $0^{\circ}, 90^{\circ}, 180^{\circ}$ and $270^{\circ}$, obstruct part of the anode's FOVs near the spokes (see Figure 6).

The elevation angle-energy response of the MINPA utilizes ion beams with energies of $10 \mathrm{eV}, 50 \mathrm{eV}, 300 \mathrm{eV}, 1 \mathrm{keV}, 3 \mathrm{keV}, 10 \mathrm{keV}$ and $20 \mathrm{keV}$. The response of a particular beam energy is obtained by sweeping the high voltages of ESA and Deflectors 1 and 2 . Figure 7 shows an example of the elevation angle-energy response of the $3 \mathrm{keV}$ ion beam for Anode 10. The horizontal axis of Figure 7a shows the two-dimensional (2D) distribution of counts versus ion energy; the vertical axis gives the elevation angle. We summarize the $2 \mathrm{D}$ count distribution over one dimension to get the reduced $1 \mathrm{D}$ count distribution along the other dimension. Figures $7 \mathrm{~b}$ and $7 \mathrm{c}$ show the $1 \mathrm{D}$ count response versus elevation angle and energy. The Gaussian function fittings (red curves in Figures $7 \mathrm{~b}$ and $7 \mathrm{c}$ ) give the elevation-angle resolution and the energy resolution, which are $5.4^{\circ}$ (FWHM) and $12.1 \%$ (FWHM), respectively.

The $k$-factor is the ratio between the selected beam energy and the high voltage applied in the ESA; it is constant at a given ESA geometry design. The solid square dots in Figure 8 show the beam energy versus the ESA high voltage presented in logarithmic scale. The black curve is the linear fit of the solid squares, and its slope (5.62) gives the average $k$-factor of the MINPA. The adjusted $R$-square value, which shows the fit accuracy, is 0.99998 .

The elevation angle selection of the ion measurement channel is determined by the high voltages applied on Deflectors 1 and 2 . Here, we define the deflection factor $R$ as follows:

$$
R=\left(V_{\mathrm{DF} 1}-V_{\mathrm{DF} 2}\right) / E_{\text {beam }}
$$

where $V_{\mathrm{DF} 1}$ and $V_{\mathrm{DF} 2}$ are the high voltages applied on Deflectors 1 and 2 , and $E_{\text {beam }}$ is the selected beam energy for the calibration. Figure 9 shows the relationship between the deflection factor $R$ and the selected elevation angle. During the flight operation, the measuring elevation angles of the ions in space are derived from the high voltages on Deflectors 1 and 2 and the ESA high voltages, according to the relationship in Figure 9.

The ion species are distinguished by their times of flight through the TOF unit. For a given ion mass and a flight distance, the time of flight depends on the residual energy (total energy minus the energy loss in the carbon foil) of the ion after passing through the start carbon foil. The higher the ion residual energy, the shorter the time of flight. Here, the total ion energy means the original incident ion energy plus the post-acceleration energy added by the TOF unit. We performed the ground calibration using seven ion species with different energies, and determined their relationships between their times of flight and their total energy. Figure 10 shows the time of flight profiles of different ion species with total ion energies of $15.3 \mathrm{keV}(0.3 \mathrm{keV}$ ion original energy +15 $\mathrm{kV}$ post-acceleration $\mathrm{HV}$ ) and $35 \mathrm{keV}$ ( $20 \mathrm{keV}$ ion original energy $+15 \mathrm{kV}$ post-acceleration $\mathrm{HV}$ ).

The geometry factor (G-factor) is an integration parameter for all the calibration characteristics described above (Wurz et al., 2007). The $G$-factor of one azimuth sector is defined by the formula below.

$$
G \cong \Delta \theta \sum_{i} \sum_{j} \Delta \varphi_{i} \frac{\Delta E_{j}}{E_{j}} \frac{C_{i j}}{l_{\text {beam }}}
$$




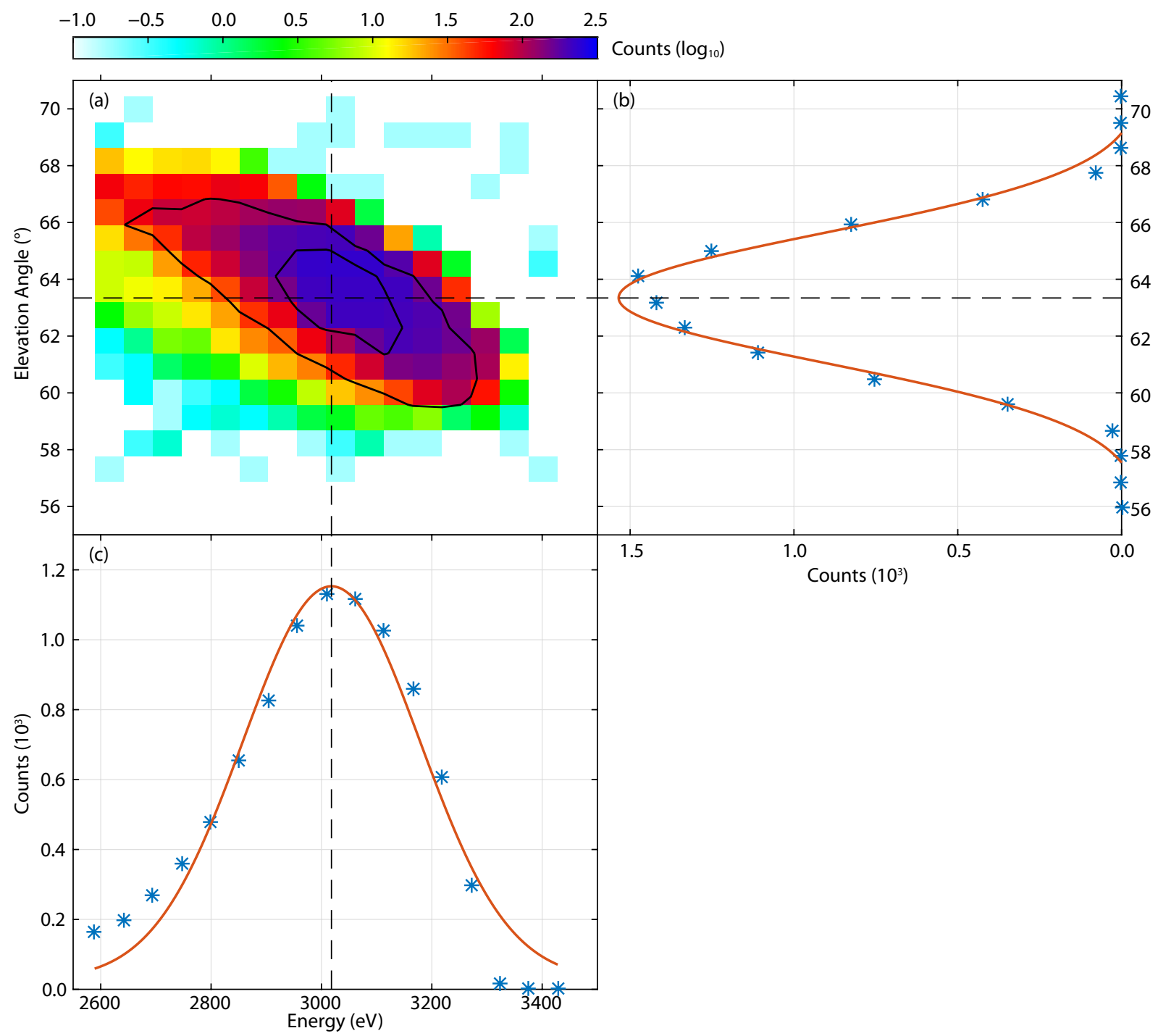

Figure 7. An example of the elevation angle-energy response of Anode 10 to the $3 \mathrm{keV}$ ion beam. (a) $2 \mathrm{D}$ count distribution plotted on a logarithmic color scale versus energy on the horizontal axis and elevation angle on the vertical axis. The reduced 1D count distribution is obtained from summing the 2D count distribution over the other dimension. The 1D reduced count distributions and the Gaussian fitting curves versus elevation angle and energy are displayed in (b) and (c), respectively.

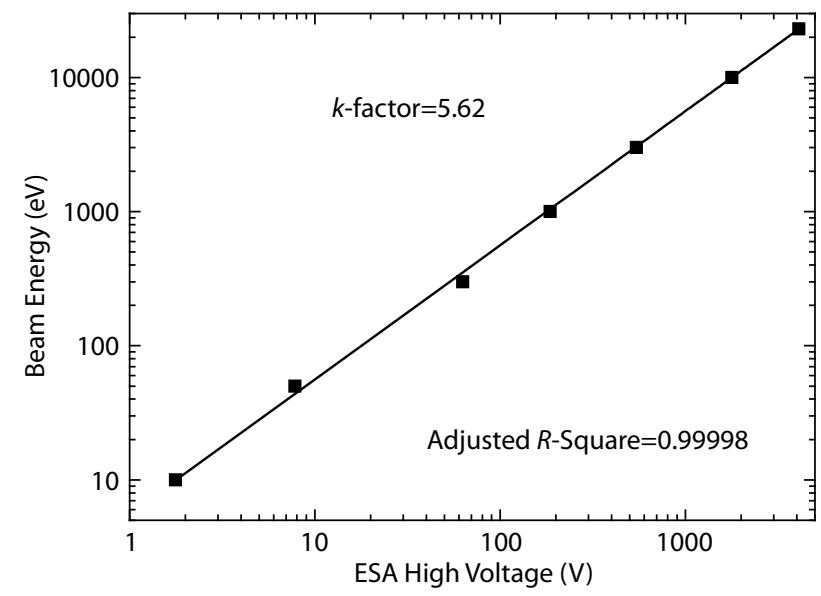

Figure 8. $k$-factor from the linear fit of the ion beam energy versus ESA high voltage. The inner ESA electrode is at a negative high voltage; the outer ESA electrode is grounded.

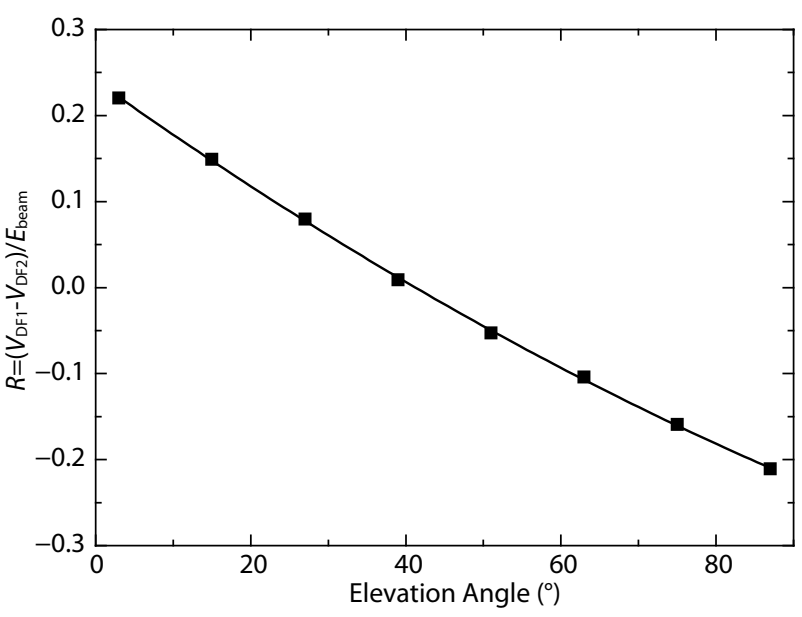

Figure 9. Relationship between the deflection factor $R$ and the elevation angle 

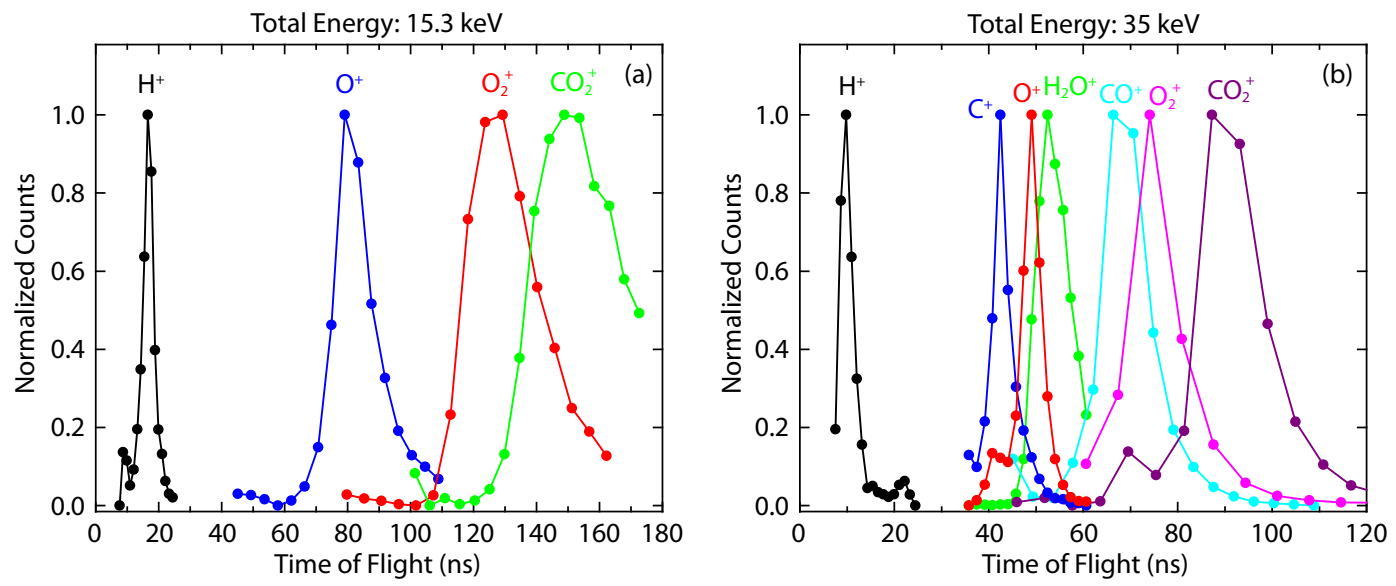

Figure 10. Time of flight profiles of different ion species with total ion energies of $15.3 \mathrm{keV}(\mathrm{a})$ and $35 \mathrm{keV}$ (b).

where $\Delta \theta$ is the azimuthal FOV of the azimuth sector, the subscripts $i$ and $j$ denote the sweeping step numbers of azimuth angle and energy, $\Delta \varphi_{i}$ and $\Delta E_{j}$ are the pass bands of elevation angle and energy at the sweeping steps of $\varphi_{i}$ and $E_{j}$, respectively, $E_{j}$ is the central energy of the energy sweeping step, $C_{i j}$ is the accumulated counts per second at the sweeping step $\left(\varphi_{i}, E_{j}\right)$, and $I_{\text {beam }}$ is the beam intensity in unit of counts per square centimeter per second. The geometry factor varies with the masses of ion species and the elevation angles. Figure 11 shows an example of the variation of the geometry factor for Anode 10 along the elevation angles for $\mathrm{O}^{+}$. The geometry factor has a maximum at $45^{\circ}$ and a minimum near $90^{\circ}$.

Table 2 summarizes the specification parameters of MINPA from the ground calibrations of the ion measurement channel.

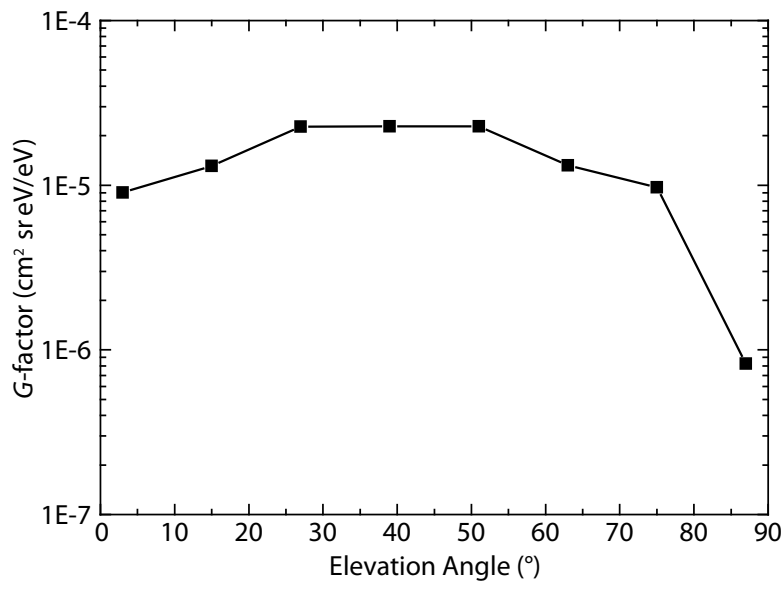

Figure 11. Geometry factor for Anode 10 along the elevation angle for $\mathrm{O}^{+}$.

\subsection{Calibration of the ENA Measurement Channel}

The ENA measurement channel was calibrated by use of the ENA beam in MEFISTO. The ENA measurement channel shares the ESA and the TOF unit with the ion measurement channel. Thus, the azimuthal response of the ENAs is treated in the same way as the ion measurement channel shown in Figure 6. The MINPA was calibrated by using $\mathrm{H}, \mathrm{He}$, and $\mathrm{O}$ ENA beams at $500 \mathrm{eV}, 1 \mathrm{keV}, 3 \mathrm{keV}$ and $3.5 \mathrm{keV}$. The ENA calibration data analysis is complicated because of the existence of sputtered ions. The $\mathrm{Al}_{2} \mathrm{O}_{3}$ coating film on the charge exchange plates develops a contamination layer (created by water vapor and some hydrocarbons left after cleaning) during calibration in the vacuum chamber. Some sputtered ions $\left(\mathrm{H}^{+}, \mathrm{C}^{+}, \mathrm{O}^{+}\right)$can be produced from the contamination layer (Fuselier et al., 2009). The sputtered ion spectrum of $\mathrm{H}^{+}, \mathrm{C}^{+}$and $\mathrm{O}^{+}$during the He beam calibration can be used as a way to estimate the sputtered ions background when analyzing the $\mathrm{H}$ and $\mathrm{O}$ calibration data.

Similar to the ion measurement calibration, the elevation angleenergy response is also critical for the ENA calibration. Figure 12 shows an example of the elevation angle-energy response of a 3 $\mathrm{keV} \mathrm{H}$ neutral beam at Anode 6. Figure 12a shows the 2D count distribution versus the $\mathrm{H}$ atom energy on the horizontal axis and elevation angle on the vertical axis; Figures $12 \mathrm{~b}$ and $12 \mathrm{c}$ show the $1 \mathrm{D}$ count response versus elevation angle and energy, respectively. The elevation-angle resolution and energy resolution are obtained from Gaussian fittings (solid curves in Figures $12 \mathrm{~b}$ and 12c), which are $9.7^{\circ}$ (FWHM) and $83.3 \%$ (FWHM), respectively.

Because the energy spread on the charge exchange plates is related to the ENA mass, the energy resolution of $O$ is different from that of $\mathrm{H}$. Figure 13 shows the energy response of a $3 \mathrm{keV} O$ beam; the energy resolution after the Gaussian fitting is 58.4\% (FWHM).

The ENAs lose part of their energy when interacting with the charge exchange plates. The fraction of energy loss also depends on the energy and mass of the ENAs. Figure 14 shows the energy losses of $\mathrm{H}$ (solid stars) and $\mathrm{O}$ (solid squares). The energy loss of $\mathrm{O}$ is higher than that of $\mathrm{H}$ for the same initial energy.

From the ENA measurement principle, the mass of the ENAs and their corresponding ionized ions should be the same during the process of charge exchanging. Thus, the mass of ENAs can be derived from the TOFs of the ionized ions produced by the charge exchange plates. Figure 15 shows the TOF profiles of the $3 \mathrm{keV} \mathrm{H}$ (solid stars) and $\mathrm{O}$ (solid squares) neutral beams. It can be seen that the TOF data allow $\mathrm{H}$ and $\mathrm{O}$ to be clearly distinguished from each other.

ENAs get ionized during their interaction with the charge ex- 
Table 2. MINPA specification parameters of the ion measurement.

\begin{tabular}{ccc}
\hline Items & Values & Comments \\
\hline ESA $k$-factor & 5.62 & ESA high voltage range: $0.5-4618 \mathrm{~V}$ \\
Energy range & $2.8 \mathrm{eV}-25.9 \mathrm{keV}$ & $12.1 \%(\mathrm{FWHM})$ \\
ESA energy resolution & $360^{\circ} \times 90^{\circ}$ & \\
Field-of-view & 16 & $\mathrm{H}^{+}$to $\mathrm{CO}_{2}{ }^{+}$are verified by calibration \\
Azimuth sectors & 16 & Adjustable $_{\text {Deflection steps }}$ \\
Resolved masses range & $1-70 \mathrm{amu}$ & ${\text { Efficiency is included; for } \mathrm{O}^{+}, \text {elevation } 45^{\circ}}^{\text {Time resolution }}$ \\
G-factor/sector & $4 \mathrm{~s}$ &
\end{tabular}

\begin{tabular}{rrrrrr}
-0.5 & 0.0 & 0.5 & 1.0 & 1.5 & 2.0 \\
\hline & 1 & & & & \\
\hline
\end{tabular}

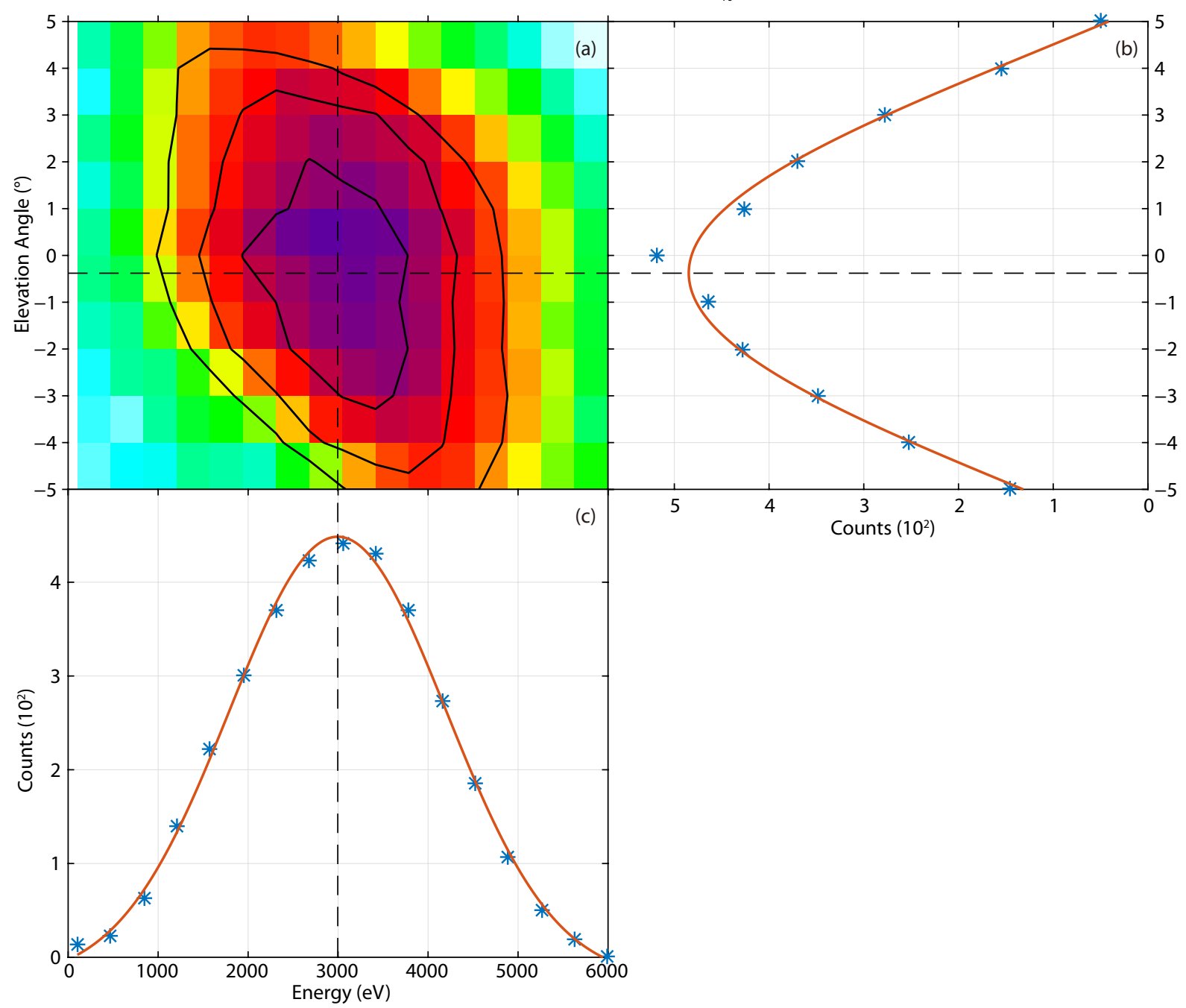

Figure 12. An example of the elevation angle-energy response of a $3 \mathrm{keV} \mathrm{H}$ neutral beam in the case of Anode 6 (same with Figure 7).

change plates, and the ionization efficiency depends significantly on the energy and mass of the ENAs. The ionization efficiencies are also included in the geometry factor calculation for different energies and ENAs species. Figure 16 shows the geometry factors of $\mathrm{H}$ and $\mathrm{O}$ of energies from $500 \mathrm{eV}$ to $3 \mathrm{keV}$; their ionization effi- ciencies are taken into account in the G-factor definition in Formula (2). The count rate detected by the MINPA is relatively low due to a low beam intensity and short accumulated slot time (170 $\mathrm{ms}$ ) for the ENA calibration. The statistics errors of the $G$-factors are presented in Figure 16. ENAs with energies below $500 \mathrm{eV}$ were 


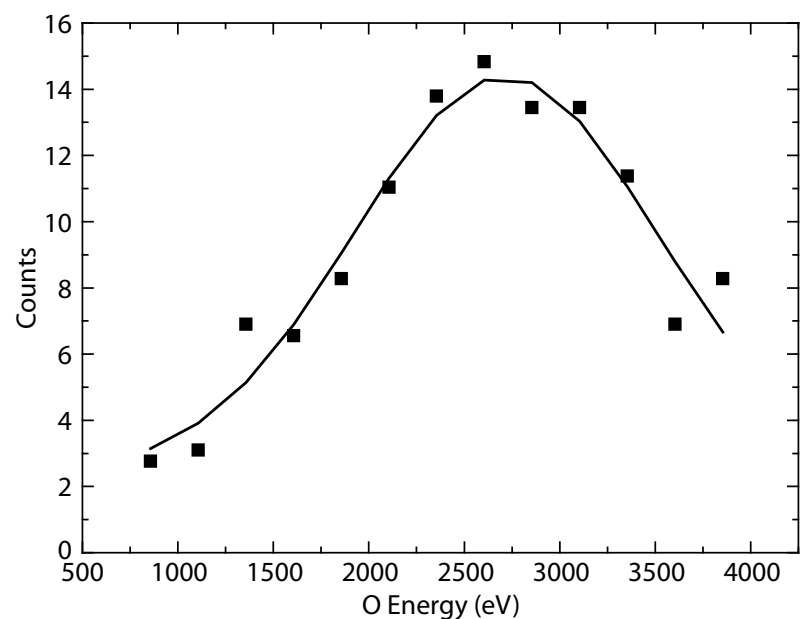

Figure 13. Energy response of $3 \mathrm{keV} \mathrm{O}$. The solid square dots show the calibration data; the solid curve denotes its Gaussian fitting.

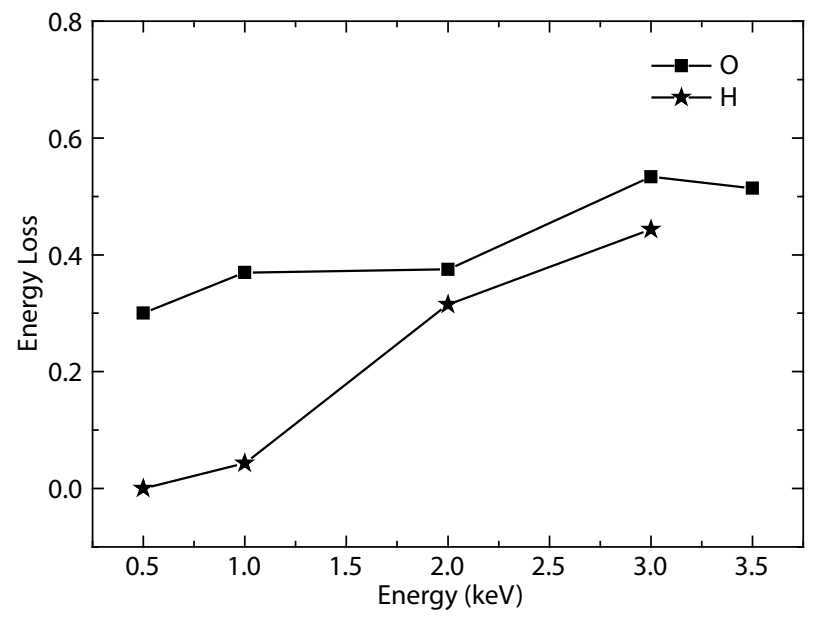

Figure 14. Energy loss of $\mathrm{H}$ and $\mathrm{O}$ as a function of the initial ENA energy. Solid stars and squares show the calibration data for $\mathrm{H}$ and $\mathrm{O}$, respectively.

not calibrated because not enough counts could be output due to the low beam intensity and short accumulated slot time available.

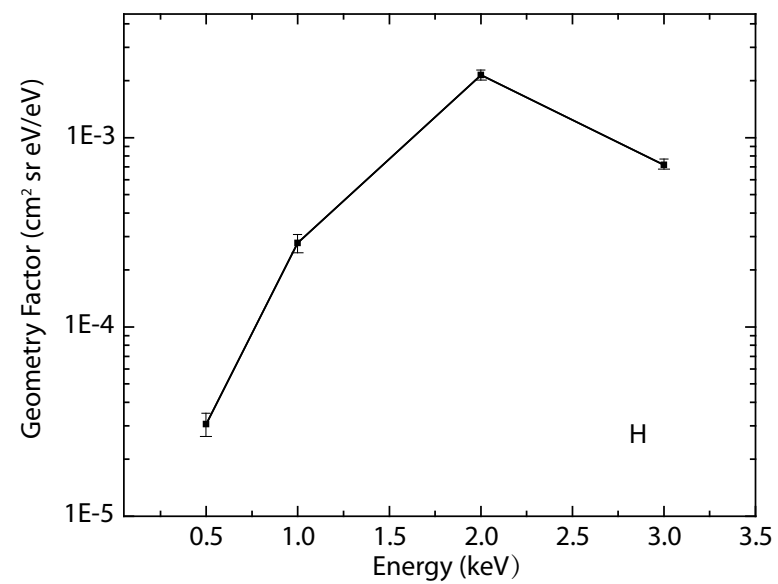

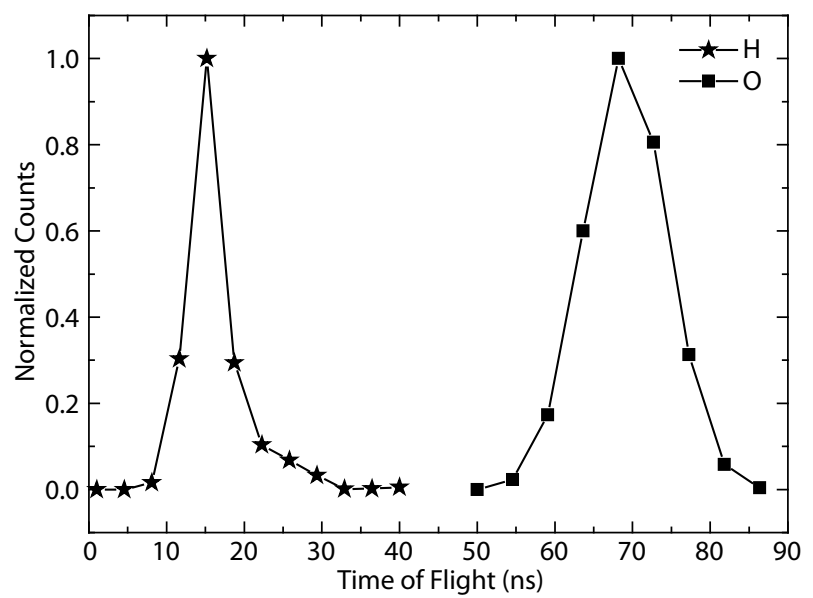

Figure 15. Time of flight profiles of the $3 \mathrm{keV} \mathrm{H}$ and $\mathrm{O}$ neutral beams. Solid stars and squares show the calibration data for $\mathrm{H}$ and $\mathrm{O}$, respectively

The corresponding geometry factors could be extrapolated for energies down to $50 \mathrm{eV}$ based on the relative ionization efficiencies measured in previous ENA instrument calibrations (Moore et al., 2000; Barabash et al., 2006; Scheer et al., 2009; Fuselier et al., 2009).

The specification parameters of the ENA measurement channel are summarized in Table 3.

\subsection{Calibration of the UV Suppression}

The MCPs used by the MINPA are also sensitive to UV light. To suppress UV light contamination, the mechanical parts in the path of the ions and ENAs are blacked to have a very high UV absorption efficiency (>98\%). The UV suppression calibration is carried out using the UV lamp in the MEFISTO chamber. The UV lamp emits nearly monochromatic light with a central wavelength of $126 \pm 5 \mathrm{~nm}$ and intensity of $3.84 \times 10^{13}$ photons $/ \mathrm{cm}^{2} / \mathrm{s}$ at the MINPA calibration location. Figure 17 shows the count rates of the 16 azimuthal anodes during the UV suppression calibration.

From the UV light detection efficiency data of the MCPs (Technical Information of MCP Assembly by Hamamatsu (Hamamatsu,

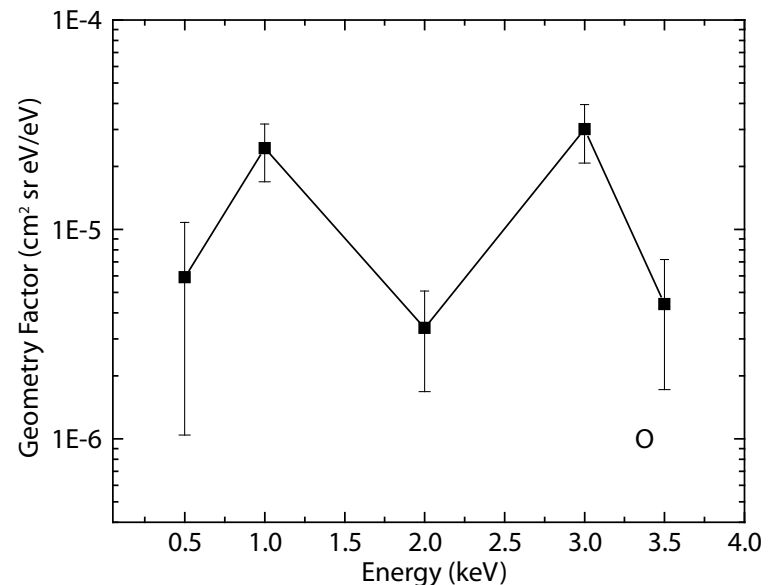

Figure 16. Geometry factors for Anode 10 versus energies of $\mathrm{H}$ and $\mathrm{O}$. The vertical bars denote the statistics errors. 
Table 3. MINPA specification parameters of the ENA measurement.

\begin{tabular}{ccc}
\hline Items & Values & comments \\
\hline Energy range & $50 \mathrm{eV}-3 \mathrm{keV}$ & Controlled by the high voltages setting \\
Energy resolution & $83.3 \%(\mathrm{FWHM})$ for $\mathrm{H} ; 58.4 \%$ (FWHM) for O & \\
Field-of-view (azimuth $\times$ elevation) & $360^{\circ} \times 9.7^{\circ}(\mathrm{FWHM})$ & 16 \\
Azimuth sectors & $1,(\geq 16$, group) amu & Adjustable \\
Resolved masses range & $4 \mathrm{~s}$ & \\
Time resolution &
\end{tabular}

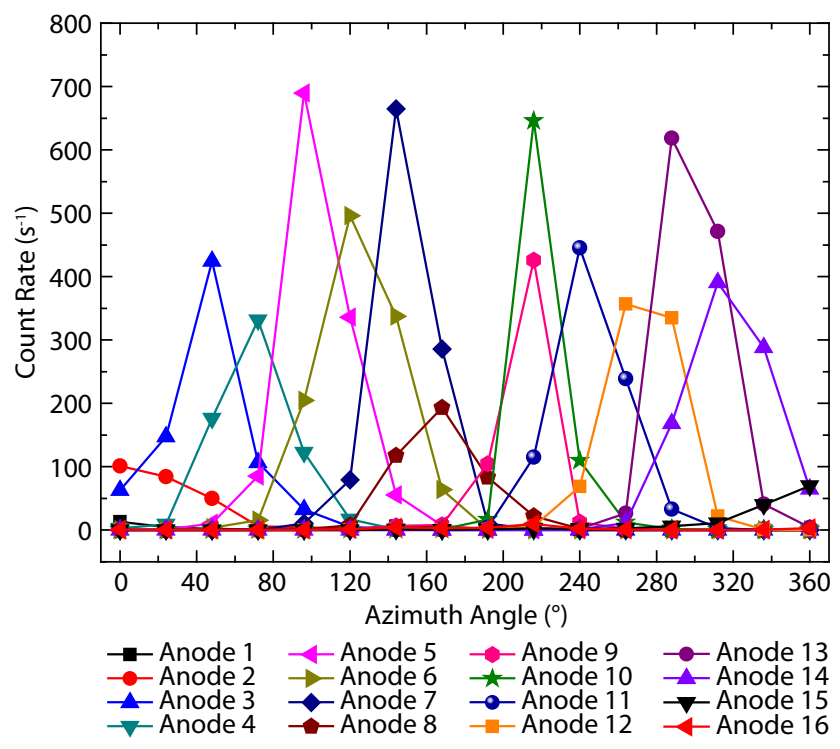

Figure 17. UV response of the 16 anodes during the calibration.

2006)), the efficiency of the UV light at $180 \mathrm{~nm}$ is approximately two orders of magnitude lower than that of the Lyman- $\alpha$ photons. Therefore, we can assume that the MCPs are sensitive only to UV light with wavelengths shorter than $180 \mathrm{~nm}$. A total real solar UV light intensity of $2 \times 10^{12}$ photons $/ \mathrm{cm}^{2} / \mathrm{s}$ with wavelengths shorter than $180 \mathrm{~nm}$ (SOLAR 2000 model) is used for the comparison analysis. Thus, the count rate on each azimuth anode due to UV contamination is a factor of 19.2 lower than the calibration response shown in Figure 17. This factor is underestimated because the MCP efficiency for UV light with wavelength longer than 150 $\mathrm{nm}$ is very low, while the irradiance above $150 \mathrm{~nm}$ accounts for approximately $60 \%$ of the total irradiance below $180 \mathrm{~nm}$.

\section{Summary}

The Mars lon and Neutral Particle Analyzer (MINPA), an integrated instrument designed for the Chinese Mars Exploration Mission, will measure the ions and ENAs in the solar wind and the Mars' magnetosphere; the acquired data will be used to study the interaction of the solar wind with the Martian atmosphere. This paper has described the design of the MINPA and its ground calibration, detailing its specifications and performance. The MINPA can measure ions with energies from $2.8 \mathrm{eV}$ to $25.9 \mathrm{keV}$ within a $360^{\circ} \times$ $90^{\circ}$ field of view, and ENAs with energies from $50 \mathrm{eV}$ to $3 \mathrm{keV}$ within a $360^{\circ} \times 9.7^{\circ}$ field of view. The ion measurement channel of the MINPA can resolve the ion species as $\mathrm{H}^{+}, \mathrm{He}^{2+}, \mathrm{He}^{+}, \mathrm{O}^{+}, \mathrm{O}_{2}{ }^{+}$, and
$\mathrm{CO}_{2}{ }^{+}$. The ENA measurement channel can resolve the $\mathrm{H}$ and $\mathrm{O}$ groups ( $\geq 16 \mathrm{amu}$ ). After the launch of the spacecraft, we will carry out an in-flight calibration to identify the performance of the MINPA so as to provide high-quality scientific data for the space science community.

\section{Acknowledgments}

The development work was funded by the Chinese National Space Administration. We acknowledge constructive suggestions from Prof. Berndt Klecker during instrument development. We thank all the MINPA team members for their very dedicated work. We acknowledge the solar UV irradiance spectrum data from the SOLAR 2000 model.

\section{References}

Barabash, S., Lundin, R., Andersson, H., Brinkfeldt, K., Grigoriev, A., Gunell, H., Holmström, M., Yamauchi, M., Asamura, K., ... Thocaven, J. J. (2006). The analyzer of space plasmas and energetic atoms (ASPERA-3) for the Mars Express Mission. Space Sci. Rev., 126(1-4), 113-164. https://doi.org/10.1007/s11214-006-9124-8

Barabash, S., Fedorov, A., Lundin, R., and Sauvaud, J. A. (2007). Martian atmospheric erosion rates. Science, 315(5811), 501-503. https://doi.org/10.1126/science.1134358

Carlson, C. W., and McFadden, J. P. (1998). Design and application of imaging plasma instruments. In R. F. Pfaff, et al. (Eds.), Measurement Techniques in Space Plasmas: Particles. Washington: AGU. https://doi.org/10.1029/GM102p0125

Carlsson, E., Fedorov, A., Barabash, S., Budnik, E., Grigoriev, A., Gunell, H., Nilsson, H., Sauvaud, J. A., Lundin, R., ... Dierker, C. (2006). Mass composition of the escaping plasma at Mars. Icarus, 182(2), 320-328. https://doi.org/10.1016/j.icarus.2005.09.020

Collier, M. R., Moore, T. E., Ogilvie, K. W., Chornay, D., Keller, J. W., Boardsen, S., Burch, J., El Marji, B., Fok, M. C., ... Wurz, P. (2001). Observations of neutral atoms from the solar wind. J. Geophys. Res. Space Phys., 106(A11), 24893-24906. https://doi.org/10.1029/2000JA000382

Dongfang, X. (2016). China's first Mars probe made its debut, calling for engineering name and logo. Space Int. (in Chinese)(9), 41-44.

Fang, X. H., Liemohn, M. W., Nagy, A. F., Luhmann, J. G., and Ma, Y. J. (2010). Escape probability of Martian atmospheric ions: Controlling effects of the electromagnetic fields. J. Geophys. Res. Space Phys., 115(A4), A04308. https://doi.org/10.1029/2009JA014929

Farmer, C. B., Davies, D. W., Holland, A. L., LaPorte, D. D., and Doms, P. E. (1977). Mars: water vapor observations from the Viking orbiters. J. Geophys. Res., 82(28), 4225-4248. https://doi.org/10.1029/JS082i028p04225

Fuselier, S. A., Bochsler, P., Chornay, D., Clark, G., Crew, G. B., Dunn, G., Ellis, S., Friedmann, T., Funsten, H. O., ... Zaffke, S. (2009). The IBEX-Lo sensor. Space Sci. Rev., 146(1-4), 117-147. https://doi.org/10.1007/s11214-009-9495-8

Futaana, Y., Barabash, S., Grigoriev, A., Holmström, M., Kallio, E., Brandt, P. C., Gunell, H., Brinkfeldt, K., Lundin, R.,... Dierker, C. (2006). First ENA observations at Mars: ENA emissions from the Martian Upper Atmosphere. 
Icarus, 182(2), 424-430. https://doi.org/10.1016/j.icarus.2005.09.019

Galli, A., Wurz, P., Kallio, E., Ekenbäck, A., Holmström, M., Barabash, S., Grigoriev, A., Futaana, Y., Fok, M. C., and Gunell, H. (2008). Tailward flow of energetic neutral atoms observed at Mars. J. Geophys. Res. Planets, 113(E12), E12012. https://doi.org/10.1029/2008JE003139

Gunell, H., Brinkfeldt, K., Holmström, M., Brandt, P., Barabash, S., Kallio, E., Ekenbäck, A., Futaana, Y., Lundin, R.,... Dierker, C. (2006). First ENA observations at Mars: Charge exchange ENAs produced in the magnetosheath. Icarus, 182(2), 431-438. https://doi.org/10.1016/j.icarus.2005.10.027

Hamamatsu. (2006). Technical information of MCP assembly. TMCP9002E01, printed in Japan.

Klumpar, D. M., Möbius, E., Kistler, L. M., Popecki, M., Hertzberg, E., Crocker, K., Granoff, M., Tang, L., Carlson, C. W., ... Hovestadt, D. (2001). The time-offlight energy, angle, mass spectrograph (Teams) experiment for Fast. Space Sci. Rev., 98(1-2), 197-219. https://doi.org/10.1023/A:1013127607414

Ledvina, S. A., Brecht, S. H., Brain, D. A., and Jakosky, B. M. (2017). Ion escape rates from Mars: Results from hybrid simulations compared to MAVEN observations. J. Geophys. Res. Space Phys., 122(8), 8391-8408. https://doi.org/10.1002/2016JA023521

Lundin, R., Zakharov, A., Pellinen, R., Barabasj, S. W., Borg, H., Dubinin, E. M., Hultqvist, B., Koskinen, H., Liede, I., and Pissarenko, N. (1990). Aspera/Phobos measurements of the ion outflow from the MARTIAN ionosphere. Geophys. Res. Lett., 17(6), 873-876. https://doi.org/10.1029/GL017i006p00873

Lundin, R., Barabash, S., and the ASPERA-3 Team. (2009). ASPERA-3: Analyser of space plasmas and energetic neutral atoms. ESA SP-1291: Mars Express-The Scientific Investigations. ESA, 199-215.

McFadden, J. P., Kortmann, O., Curtis, D., Dalton, G., Johnson, G., Abiad, R., Sterling, R., Hatch, K., Berg, P., ... Jakosky, B. (2015). MAVEN suprathermal and thermal ion compostion (STATIC) Instrument. Space Sci. Rev., 195(1-4), 199-256. https://doi.org/10.1007/s11214-015-0175-6

McKenna-Lawlor, S., Li, L., Barabash, S., Kudela, K., Balaz, J., Strharsky, I., Brinkfeldt, K., Gunell, H., Shen, C., ... Dandouras, I. (2005). The NUADU experiment on TC-2 and the first Energetic Neutral Atom (ENA) images recorded by this instrument. Ann. Geophys., 23(8), 2825-2849. https://doi.org/10.5194/angeo-23-2825-2005

Mitchell, D. L., Lin, R. P., Mazelle, C., Rème, H., Cloutier, P. A., Connerney, J. E. P., Acuña, M. H., and Ness, N. F. (2001). Probing Mars' crustal magnetic field and ionosphere with the MGS Electron Reflectometer. J. Geophys. Res. Planets, 106(E10), 23419-23427. https://doi.org/10.1029/2000JE001435

Moore, T. E., Chornay, D. J., Collier, M. R., Herrero, F. A., Johnson, J., Johnson, M. A., Keller, J. W., Laudadio, J. F., Lobell, J. F., ... Wilson, G. R. (2000). The lowenergy neutral atom imager for IMAGE. Space Sci. Rev., 91(1-2), 155-195. https://doi.org/10.1023/A:1005211509003

Moore, T. E., Collier, M. R., Burch, J. L., Chornay, D. J., Fuselier, S. A., Ghielmetti, A.
G., Giles, B. L., Hamilton, D. C., Herrero, F. A.,... Wurz, P. (2001). Low energy neutral atoms in the magnetosphere. Geophys. Res. Lett., 28(6), 1143-1146. https://doi.org/10.1029/2000GL012500

Neugebauer, M., and Snyder, C. W. (1962). Solar plasma experiment. Science, 138(3545), 1095-1097. https://doi.org/10.1126/science.138.3545.1095-a

Ramstad, R., Futaana, Y., Barabash, S., Nilsson, H., del Campo B, S. M., Lundin, R., and Schwingenschuh, K. (2013). Phobos 2/ASPERA data revisited: Planetary ion escape rate from Mars near the 1989 solar maximum. Geophys. Res. Lett., 40(3), 477-481. https://doi.org/10.1002/grl.50149

Rème, H., Bosqued, J. M., Sauvaud, J. A., Cros, A., Dandouras, J., Aoustin, C., Bouyssou, J., Camus, T., Cuvilo, J., ... Balsiger, H. (1997). The cluster ion spectrometry (CIS) experiment. Space Sci. Rev., 79(1-2), 303-350. https://doi.org/10.1023/A:1004929816409

Rème, H., Aoustin, C., Bosqued, J. M., Dandouras, I., Lavraud, B., Sauvaud, J. A., Barthe, A., Bouyssou, J., Camus, T., ... Sonnerup, B. (2001). First multispacecraft ion measurements in and near the Earth's magnetosphere with the identical Cluster ion spectrometry (CIS) experiment. Ann. Geophys., 19(10-12), 1303-1354. https://doi.org/10.5194/angeo-19-1303-2001

Rème, H., Dandouras, I., Aoustin, C., Bosqued, J. M., Sauvaud, J. A., Vallat, C., Escoubet, P., Cao, J. B., Shi, J., ... the HIA Team. (2005). The HIA instrument on board the Tan Ce 1 Double Star near-equatorial spacecraft and its first results. Ann. Geophys., 23(8), 2757-2774. https://doi.org/10.5194/angeo-232757-2005

Riedo, A. (2010). Optimization of the detection-technique for energetic neutral atoms for the BepiColombo mission [Ph. D. thesis]. Bern: University of Bern.

Scheer, J. A., Wahlström, P., and Wurz, P. (2009). Scattering of light molecules from thin $\mathrm{Al}_{2} \mathrm{O}_{3}$ films. Nucl. Instrum. Methods Phys. Res. B, 267(16), 2571-2574. https://doi.org/10.1016/j.nimb.2009.05.016

Verigin, M. I., Gringauz, K. I., Kotova, G. A., Shutte, N. M., Rosenbauer, H., Livi, S., Richter, A. K., Riedler, W., Schwingenschuh, K., and Szegö, K. (1991). On the problem of the Martian atmosphere dissipation: Phobos: 2 TAUS Spectrometer results. J. Geophys. Res. Space Phys., 96(A11), 19315-19320. https://doi.org/10.1029/90JA02561

Wieser, M., and Wurz, P. (2005). Production of a $10 \mathrm{eV-1000} \mathrm{eV} \mathrm{neutral} \mathrm{particle}$ beam using surface neutralization. Meas. Sci. Technol., 16(12), 2511-2516. https://doi.org/10.1088/0957-0233/16/12/016

Wurz, P. (2000). Detection of energetic neutral particles. In K. Scherer, et al. (Eds.), The Outer Heliosphere: Beyond the Planets (pp. 251-288).Germany: Copernicus Gesellschaft e.V., Katlenburg-Lindau.

Wurz, P., Balogh, A., Coffey, V., Dichter, B. K., Kasprzak, W. T., Lazarus, A. J., Lennartsson, W., and McFadden, J. P. (2007). Calibration techniques. In: M. Wüest, et al. (Eds.), Calibration of Particle Instruments in Space Physics (pp. 117-276). Noordwijk: ESA Communications.

Ye, P. J., Sun, Z. Z., Rao, W., and Meng, L. Z. (2017). Mission overview and key technologies of the first Mars probe of China. Sci. China Technol. Sci., 60(5), 649-557. https://doi.org/10.1007/s11431-016-9035-5 